COMMENT. Long-term VPA and LTG therapy, especially in combination, is associated with short stature, low bone mineral density, and reduced bone formation. These effects on growth and bone metabolism are correlated with reduced physical activity. Although a specific effect of the anticonvulsant medication on growth and bone mass is unproven, further studies are indicated.

\title{
CELIAC DISEASE AND LOCALIZATION-RELATED EPILEPSIES
}

The incidence of silent ceilac disease (CD) in children with idiopathic localization epilepsies and the indications for routine $C D$ screening were determined in a study of 72 patients ( 31 girls and 41 boys; mean age 12.6 years; age at onset 6.4 years) observed over a 5 year period at the Institute of Neurology and Gastroenterology, University Magna Graecia of Catanzaro, Italy. The enzymelinked immunosorbent assay (ELISA) for antigliadin antibodies (AGA) and the immunofluorescent undirected test for antiendomysium antibodies (AEA) were used to confirm a diagnosis of CD. ELISA has $>96 \%$ sensitivity and $97 \%$ specificity for IgA-AGA and IgG-AGA antibodies. AEA is a more specific but less sensitive test than AGA. Twenty five patients had childhood partial epilepsy with occipital

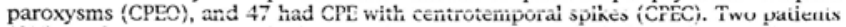
(8\%) in the CPEO group had antiendomysium immunoglobulin (Ig) A antibodies, and their jejunal biopsies showed atrophy of the villi and hyperplasia of crypts, confirming the diagnosis of CD. Brain CT was normal in one and showed occipital cortical-subcortical calcifications in the other. Treatment with a gluten-free diet was followed by seizure remission, and no calcifications developed in the patient with a normal CT after 3 year follow up. None of the patients with CPEC had positive antibody tests for CD. (Labate A, Gambardella A, Messina D et al. Silent celiac disease in patients with childhood localization-related epilepsies. Epilepsia Sept 2001;42:1153-1155). (Reprints: Dr A Gambardella, Cattedra ed UO di Neurologia, Universita degli studi Magna Graecia, Policlinico Mater Domini, Via Tommaso Campanella, 88100 Catanzaro, Italy).

COMMENT. Celiac disease screening is recommended in patients with childhood partial epilepsy with occipital paroxysms (CPEO). Early diagnosis of CD and dietary intervention may reverse the tendency to seizures and the development of brain calcifications. Routine screening for $\mathrm{CD}$ is not indicated in patients with infantile extraoccipital seizures. A new diagnostic test for CD using human recombinant tissue transglutaminase (TACA) may he mere sencitive in the diagnosis of silent forms of CD (Sblattero D et al. Am / Gastroenterol 2000;95:1253-7, cited by the authors).

\section{BENIGN OCCIPITAL SEIZURE SUSCEPTIBILITY SYNDROME}

Thirty seven chidren who met the diagnostic criteria for early-onset benign occipital seizure susceptibility syndrome (BOSSS) were followed prospectively for more than two years at the Tokyo Women's Medical University and Doaikai Hospital, Tokyo, Japan. This diagnosis accounted for $11.7 \%$ of children with localization-related epilepsy, who developed a first seizure before 13 years of age, and presented at one of two seizure clinics between 1989 and 1998. Diagnostic criteria employed were as follows: 1) normal development before seizure onset; 2) onset 1 to 8 years of age; 3) normal MRI or CT; 4) ictal vomiting and tonic eyedeviations; 5) normal EEG background activity with or without epileptic foci regardless of location. The Panayiotopoulos criterion of occipital EEG paroxysms was excluded, since EEG foci often shift to centrotemporal or frontal regions (Ferrie et al 1997; Oguni et al 1999). In this series initial EEG spike foci were occipital in 26 (70\%), 17 of whom later revealed a shift in location, extraoccipital 
in $19 \%$, and were absent in $11 \%$. At final follow-up, only $43 \%$ foci were occipital, $46 \%$ extraoccipital, and absent in $11 \%$. A family history of epilepsy was identified in 12 patients (32\%), including a family with 2 children having BOSSS. Febrile convulsions preceded onset of BOSSS in 14 (38\%). Total number of BOSSS seizures was 1 - 27 (median 5). Status epilepticus occurred in 22 (59\%). Remission for longer than 2 years was achieved in $28(76 \%)$; and $80 \%$ of these remitted by 3 years after onset. Antiepileptic drugs had been discontinued in 21 (57\%). Fifteen children (40\%) with recurrent prolonged seizures, initially refractory to AEDs, had an earlier onset and more frequent complications. Seizures remitted by 12 years in all cases. (Oguni H, Hayashi K, Funatsuka M, Osawa M. Study on earlyonset benign occipital seizure susceptibility syndrome. Pediatr Neurol October 2001;25:312-318). (Respond: Dr Hirokazu Oguni, Department of Pediatrics, Tokyo Women's Medical University, 8-1 Kawada-cho, Shinjuku-ku, Tokyo 162, Japan).

COMMENT. The clinical features of early-onset BOSSS vary from a single or occasional seizure to cases with recurrent prolonged seizures, initially AED resistant, and even status epilepticus in more than half the patients, despite ultimate remission by 12 yeare of age. Datients with prolenged scizurcs, initially resistant to $\mathrm{AED}$, are recognized by an earlier onset and more frequent complications (prematurity with asphyxia, neonatal seizure, borderline IQ). The authors propose that the term occipital be removed from the terminology, since patients meeting all other criteria for BOSSS may have extraoccipital EEG spike foci or sometimes no EEG epileptic foci.

\section{OPTICAL FILTERS FOR TV-INDUCED PHOTOSENSITIVE EPILEPSY}

Two different types of optical filters were tested in 20 photosensitive epileptic patients for ability to inhibit photoparoxysmal response (PPR) and seizures, in a study at Gifu Prefectural Gifu Hospital, Japan. One filter reflected long-wavelength red light selectively (wavelength dependent) and the other absorbed light in the visible spectrum evenly (quantity of light-dependent). Both filters individually provided insufficient inhibition of PPR $(<50 \%)$ while using conventional strobe intermittent photic stimulation (IPS), whereas compound filters (both types) inhibited PPR by $90 \%$ for IPS and $95 \%$ for photic stimulation using cathode ray tubes (CRT). Compound optical filters do not block the chromaticity of emissions from TV-CRT, and may be used to prevent TV-induced seizures in nhotosensitive nersons. (Takahashi $Y$ Sato $T$, fotn $K$, et al. Dotical filtore inhibiting teievision-induced photosensitive seizures. Neurology November ( 2 of 2) 2001;57:1767-1773). (Reprints: Dr Yukitoshi Takahashi, Department of Pediatrics, Gifu Prefectural Gifu Hospital, 4-6-1 Noishiki Gifu 500-8717, Japan).

COMMENT. The use of optical filters to prevent photosensitive epilepsy is not a novel suggestion. Glasses with a neutral filter were introduced some 50 years ago by Bickford RG et al (Am I Dis Children 1953;86:170) at the Mayo Clinic. The use of compound filters may lessen the risk of seizures or epilepsy onset in persons with latent photosensitivity when viewing TV or using personal computers. Photosensitivity is a common problem affecting 4 to $9 \%$ of the population. It has an autosomal dominant inheritance and a strong age-dependent penetrance. In recent years, the incidence of photosensitive reflex epilepsy has escalated as an environmental hazard, mainly associated with the introduction of electronic devices, particularly video games. A mass seizure outbreak in Japan was caused by an animated TV program for children, 'Pocket Monsters'. (Erba G. Preventing seizures from "Pocket Monsters." A way to control reflex epilepsy. Editorial, Neurology Nov (2 of 2) 2001;57:1747-1748). The onus for prevention should be 